\title{
Three-dimensional modeling of shape-memory materials
}

\author{
F. Auricchio
}

Dipartimento di Meccanica Strutturale, Università di Pavia, Via Ferrata 1, 27100 Pavia, Italy

\begin{abstract}
The paper describes a model able to reproduce through a phenomenological approach some of the main macroscopic features of shape-memory materials for three-dimensional states of stress. In particular, we consider in details the case of superelastic response, giving insights on how extending the model to include the shape-memory effect.

The model is framed on a large deformation context and it based on the introduction of a scalar internal variable - the single-variant martensite fraction - and on a tensorial internal variable measuring the single-variant martensite orientation.
\end{abstract}

\section{A THREE-DIMENSIONAL MODEL}

The goal of the present paper is to give an overview of a possible approach for the development of three-dimensional constitutive models able to reproduce at the macroscopic level some basic features of shape memory materials. In particular, the model described here derives from the one discussed in [1].

\subsection{Control and internal variables}

We assume the deformation gradient, $\boldsymbol{F}$, as the control variable and, consistently with a large part of the literature on finite-deformation inelastic models $[2,3]$, we introduce a multiplicative decomposition of $\boldsymbol{F}$ in the form $\boldsymbol{F}=\boldsymbol{F}^{e} \boldsymbol{F}^{t r}$, where $\boldsymbol{F}^{e}$ is the elastic part and $\boldsymbol{F}^{t r}$ is the inelastic part, that is the quota of the deformation gradient related to the phase transition.

So far, the multiplicative split introduced in Equation is only formal, in the sense that $\boldsymbol{F}^{\text {tr }}$ can be defined as the quota of the deformation gradient depurated by the elastic contribution [that is, $\left.\boldsymbol{F}^{t r}=\left(\boldsymbol{F}^{e}\right)^{-1} \boldsymbol{F}\right]$ and it needs to be detailed.

\subsection{Elastic constitutive equation}

Assuming an isotropic elastic response, the Kirchhoff stress $\tau$ and the elastic left Cauchy-Green tensor $b^{e}$, defined as: $\boldsymbol{b}^{e}=\boldsymbol{F}^{e} \boldsymbol{F}^{e, T}$, share the same principal directions. Accordingly, the following spectral decompositions can be introduced [4]:

$$
\boldsymbol{\tau}=\sum_{A=1}^{3} \tau_{A} \quad n^{A} \otimes n^{A} \quad, \quad \boldsymbol{t}=\sum_{A=1}^{3} \quad t_{A} \quad n^{A} \otimes n^{A} \quad, \quad b^{e}=\sum_{A=1}^{3}\left(\lambda_{A}^{e}\right)^{2} n^{A} \otimes n^{A}
$$

with $\lambda_{A}^{e}$ the elastic principal stretches (or simply the elastic stretches) and $t$ the deviatoric part of $\tau$, according to the relation:

$$
\tau=p 1+t
$$


where 1 is the second-order identity tensor, $p$ is the pressure, defined as $p=\operatorname{tr}(\tau) / 3$, and $\operatorname{tr}()$ is the trace operator.

Due to the isotropy assumption, the material free-energy $\psi$ is an isotropic function of $b^{e}$, hence, it can be expressed in terms of $\left(\lambda_{A}^{e}\right)^{2}$ :

$$
\psi=\psi\left[\left(\lambda_{A}^{e}\right)^{2}\right]
$$

The Kirchhoff stress components would then be given by:

$$
\tau_{A}=2 \frac{\partial \psi}{\partial\left[\left(\lambda_{A}^{e}\right)^{2}\right]}\left(\lambda_{A}^{e}\right)^{2}
$$

Introducing the elastic volume $J^{e}=\lambda_{1}^{e} \lambda_{2}^{e} \lambda_{3}^{e}$ and the deviatoric elastic stretches $\bar{\lambda}_{A}^{e}=\left(J^{e}\right)^{-\frac{1}{3}} \lambda_{A}^{e}$, as well as the corresponding logarithmic quantities, $\theta^{e}=\log \left(J^{e}\right)$ and $e_{A}^{e}=\log \left(\bar{\lambda}_{A}^{e}\right)$, we assume a free-energy uncoupled in a deviatoric and a volumetric contribution, as follows:

$$
\psi=U\left(\theta^{e}\right)+W\left(e_{A}^{e}\right)=\frac{1}{2} K\left[\theta^{e}\right]^{2}+G \sum_{A=1}^{3}\left[e_{A}^{e}\right]^{2}
$$

with $K$ and $G$ the bulk and the shear elastic moduli, respectively. Hence, exploiting Equation 4 and recalling Equation 2, we deduce that:

$$
\tau_{A}=p+t_{A}
$$

with:

$$
p=K \theta^{e} \quad, \quad t_{A}=2 G e_{A}^{e}
$$

\subsection{Strain decomposition}

Introducing the transformation left Cauchy-Green tensor $\boldsymbol{b}^{t r}=\boldsymbol{F}^{t r} \boldsymbol{F}^{t r, T}$ and assuming for $\boldsymbol{b}^{t r}$ the same spectral decomposition as for $\boldsymbol{\tau}$ and $\boldsymbol{b}^{e}$ :

$$
\boldsymbol{b}^{t r}=\sum_{A=1}^{3}\left(\lambda_{A}^{t r}\right)^{2} \boldsymbol{n}^{A} \otimes \boldsymbol{n}^{A}
$$

we have that:

$$
\boldsymbol{b}=\boldsymbol{F} \boldsymbol{F}^{\boldsymbol{T}}=\boldsymbol{b}^{e} \boldsymbol{b}^{t r}=\boldsymbol{b}^{t r} \boldsymbol{b}^{e} \quad \text { with } \quad \boldsymbol{b}=\sum_{A=1}^{3}\left(\lambda_{A}\right)^{2} \boldsymbol{n}^{A} \otimes \boldsymbol{n}^{A}
$$

Introducing the logarithmic strains:

$$
\epsilon_{A}=\log \left(\lambda_{A}\right) \quad, \quad \epsilon_{A}^{e}=\log \left(\lambda_{A}^{e}\right), \epsilon_{A}^{t r}=\log \left(\lambda_{A}^{t r}\right)
$$

we get an additive logarithmic strain decomposition:

$$
\epsilon_{A}=\epsilon_{A}^{e}+\epsilon_{A}^{t r}
$$

which can be rewritten in terms of the volumetric and deviatoric quantities as follows:

$$
\theta=\theta^{e}+\theta^{t r} \quad, \quad e_{A}=e_{A}^{e}+e_{A}^{t r}
$$

with:

$$
\begin{aligned}
& \theta=\log (J) \quad, \quad e_{A}=\log \left(\bar{\lambda}_{A}\right) \\
& \theta^{t r}=\log \left(J^{t r}\right) \quad, \quad e_{A}^{t r}=\log \left(\bar{\lambda}_{A}^{t r}\right) \\
& J=\lambda_{1} \lambda_{2} \lambda_{3} \quad, \quad \bar{\lambda}_{A}=(J)^{-\frac{1}{3}} \lambda_{A}
\end{aligned}
$$


Finally, we set:

$$
\epsilon_{A}^{t r}=\epsilon_{L} \xi_{S} M_{A}
$$

where: $\epsilon_{L}$ is a scalar material parameter representing the maximum deformation, obtainable only by detwinning of the multiple-variant martensite; $\xi_{S}$ is the single variant martensite fraction; $M_{A}$ are quantities taking into account the martensite orientation.

At this point, to complete the model description, we need to specify proper constitutive equations for the martensite fraction, $\xi_{S}$, and for the martensite orientation factors, $M_{A}$.

\subsection{Martensite fraction evolution}

To model the evolution of the martensite fraction evolution, we consider two phase transformations: the conversion of austenite into martensite $(A \rightarrow S)$ and the conversion of martensite into austenite $(S \rightarrow A)$. To describe a possible phase-transformation pressure-dependence, we introduce a Drucker-Prager-type loading function:

$$
F(\tau)=\|t\|+3 \alpha p \quad \text { with } \quad\|t\|=\left[\sum_{A=1}^{3}\left(t_{A}\right)^{2}\right]^{1 / 2}
$$

and $\alpha$ being a material parameter. Indicating variations in time with a superposed dot, we assume the following linear forms for the evolution of $\xi_{S}$ :

$$
\begin{aligned}
& \xi_{S}=-H^{A S}\left(1-\xi_{S}\right) \frac{\dot{F}}{F-R_{f}^{A S}} \\
& \dot{\xi}_{S}=H^{S A} \quad \xi_{S} \frac{\dot{F}}{F-R_{f}^{S A}}
\end{aligned}
$$

where $R_{f}^{A S}$ and $R_{f}^{S A}$ represent the values of the loading function at the end of the evolution processes, while the quantities $H^{A S}$ and $H^{S A}$ embed the phase-transformation activation conditions. In particular, $H^{A S}$ and $H^{S A}$ are defined by the relations:

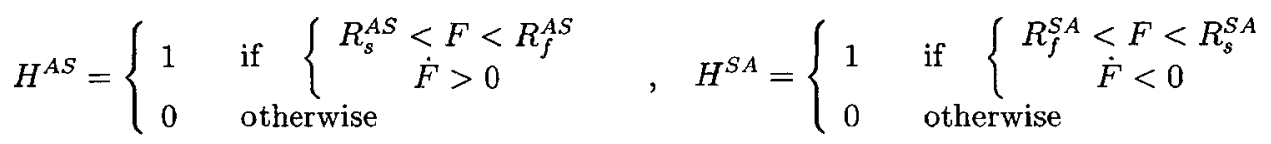

where $R_{s}^{A S}$ and $R_{s}^{S A}$ represent the values of the loading function at the beginning of the evolution processes. Possible forms for $R_{s}^{A S}, R_{f}^{A S}, R_{s}^{S A}, R_{f}^{S A}$ are:

$$
\begin{array}{ll}
R_{s}^{A S}=\left[\sigma_{s}^{A S}\left(\sqrt{\frac{2}{3}}+\alpha\right)\right], & R_{s}^{S A}=\left[\sigma_{s}^{S A}\left(\sqrt{\frac{2}{3}}+\alpha\right)\right] \\
R_{f}^{A S}=\left[\sigma_{f}^{A S}\left(\sqrt{\frac{2}{3}}+\alpha\right)\right] \quad, \quad R_{f}^{S A}=\left[\sigma_{f}^{S A}\left(\sqrt{\frac{2}{3}}+\alpha\right)\right]
\end{array}
$$

with $\sigma_{s}^{A S}, \sigma_{f}^{A S}, \sigma_{s}^{S A}, \sigma_{f}^{S A}$ material constants, which may functionally depend on the temperature.

\subsection{Martensite orientation evolution}

To specify the evolution for the orientation factors $M_{A}$, we distinguish two cases, depending if we are interested in modeling only the superelastic effect or both the superelastic and the shape-memory effect. 
In the first case we may assume that the material internal energy is such to always permit an alignment of the martensite along the the stress direction. Accordingly, introducing the quantities:

$$
N_{A}=\left.N\right|_{A}=\left.\left[\frac{\partial F}{\partial \tau}\right]\right|_{A}=n+\left.\alpha \mathbf{1}\right|_{A}=n_{A}+\alpha \quad \text { with } \quad n_{A}=\frac{t_{A}}{\|t\|}
$$

we may set $M_{A}=N_{A}$, and, combining Equations 7, 12 and 14, we get:

$$
p=K\left(\theta-3 \alpha \epsilon_{L} \xi_{S}\right) \quad, \quad t_{A}=2 G\left(e_{A}-\epsilon_{L} \xi_{S} n_{A}\right)
$$

On the other hand, if we are interested in describing both the superelastic and the shape memory effect, we cannot assume that the material internal energy always permits an alignment of the martensite along the stress directions. However, assuming a form for the orientation factors consistent with the loading function, that is of the type:

$$
M_{A}=n_{A}+\alpha \quad \text { with } \quad \sum_{A=1}^{3} M_{A}=3 \alpha
$$

we can again introduce the following relations:

$$
p=K\left(\theta-3 \alpha \epsilon_{L} \xi_{S}\right) \quad, \quad t_{A}=2 G\left(e_{A}-\epsilon_{L} \xi_{S} m_{A}\right)
$$

which are similar to the ones valid for the superelastic range. At this stage proper evolutionary equations for the $m_{A}$ factors need to be proposed, as it will be detailed in forthcoming works.

\section{NUMERICAL EXAMPLES}

To verify to properties of the proposed model, we present some numerical simulations, relative to the superelastic effect. The numerical simulations are obtained implementing the constitutive model in a finite element code, as described in Reference [5]. For the simulations, we choose the following material properties:

$$
\begin{array}{ccc}
E=5 \times 10^{4} \mathrm{MPa} & , \quad \sigma_{s}^{A S,+}=500 \mathrm{MPa} \quad, \quad \sigma_{f}^{A S,+}=500 \mathrm{MPa} \\
\sigma_{s}^{S A,+}=300 \mathrm{MPa} & , \quad \sigma_{f}^{S A,+}=300 \mathrm{MPa} \quad, \quad \sigma_{s}^{A S S_{1}-}=700 \mathrm{MPa} \\
\nu=0.3 \quad, \quad \epsilon_{L}=0.07 &
\end{array}
$$

noting that, given $\sigma_{s}^{A S,+}$ and $\sigma_{s}^{A S,-}$, the parameter $\alpha$ can be computed as follows:

$$
\alpha=\sqrt{\frac{2}{3}} \frac{\sigma_{s}^{A S,-}-\sigma_{s}^{A S,+}}{\sigma_{s}^{A S,-}+\sigma_{s}^{A S,+}}
$$

\subsection{One-dimensional tests}

We consider a cubic specimen with side equal to $1 \mathrm{~mm}$, loaded controlling the displacement in a direction parallel to one specimen side. The boundary conditions are set to reproduce a uniaxial state of stress during the loading. We consider four loading histories described in Table 1 in terms of a time scale, which however should be considered simply as a pseudo-time variable, being the model time-independent. Moreover, we observe that:

- Load 1 and Load 2 are the same but they are solved with two different time increments, a smaller one $(\mathrm{dt}=0.02 \mathrm{sec}$.) and a larger one $(\mathrm{dt}=0.2 \mathrm{sec}$.$) , respectively.$ 
Table 1: One-dimensional tests: loading histories. Four different loading histories are considered.

\begin{tabular}{|cc|cc|cc|cc|}
\hline \multicolumn{2}{|c|}{ Load 1 } & \multicolumn{2}{c|}{ Load 2 } & \multicolumn{2}{c|}{ Load 3 } & \multicolumn{2}{c|}{ Load 4 } \\
\hline time & displ. & time & displ. & time & displ. & time & displ. \\
{$[\mathrm{sec}]$} & {$[\mathrm{mm}]$} & {$[\mathrm{sec}]$} & {$[\mathrm{mm}]$} & {$[\mathrm{sec}]$} & {$[\mathrm{mm}]$} & {$[\mathrm{sec}]$} & {$[\mathrm{mm}]$} \\
\hline 0 & 0.0 & 0 & 0.0 & 0 & 0.0 & 0 & 0.0 \\
1 & 1.0 & 1 & 1.0 & 1 & 0.9 & 1 & 1.1 \\
3 & -1.0 & 3 & -1.0 & 3 & -1.1 & 3 & -0.9 \\
5 & -1.0 & 5 & 1.0 & 5 & 0.9 & 5 & -1.1 \\
\hline
\end{tabular}

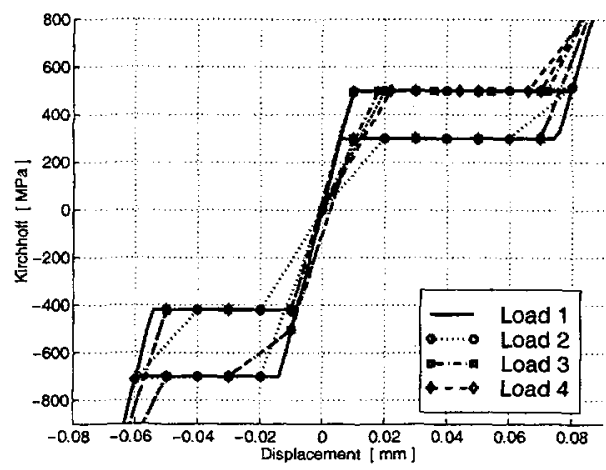

Figure 1: One-dimensional test. Kirchhoff stress versus displacement.

- Load 3 and Load 4 differ from Load 1 and Load 2 only in the peak values. Both Load 3 and Load 4 are solved with the larger time increment $(\mathrm{dt}=0.2 \mathrm{sec}$.).

For each simulation we report the response in terms of Kirchhoff stress versus displacement (Figure 1), presenting the plot only for the area of major interest (i.e. the ones where it is possible to observe the phase transformations). The solution relative to Load 1 (i.e. the case integrated with $\mathrm{dt}=0.02 \mathrm{sec}$ ) is reported with a continuous line and no symbols, while the solutions relative to the other loading cases (i.e. the cases integrated with $\mathrm{dt}=0.2 \mathrm{sec}$ ) are reported with lines and symbols (dot lines and circles for Load 2, dash-dot lines and crosses for Load 3, dash lines and diamonds for Load 4). In particular, we wish to note that for the last three loading cases there is a correspondence between symbols and instants at which the discrete solutions are computed.

For all the cases studied, it is possible to observe the optimal placements of the time-discrete solutions despite the quite large time-integration step adopted for the numerical solutions.

\subsection{An orthodontic archwire}

To test the proposed algorithm in a more complex situation, we consider the simulation of the orthodontic archwire, sketched in Figure 2. Moreover, we assume that the archwire is made out of a wire with rectangular $0.635 \mathrm{~mm} \times 0.432 \mathrm{~mm}$ cross-section.

The archwire is loaded imposing outward displacements, equal in magnitude, on sections $\mathrm{A}$ and B (Figure 2). In particular, starting from the undeformed configuration, we reach a maximum displacement of $7 \mathrm{~mm}$ in $7 \mathrm{sec}$, then bringing back to zero the imposed displacement in other $7 \mathrm{sec}$.

Due to geometric and loading symmetry considerations, in the analysis we consider only half of the archwire, introducing proper boundary conditions on the C-D plane to reproduce the symmetry conditions (Figure 2). In particular, Figure 3 shows the mesh in the undeformed configuration and 


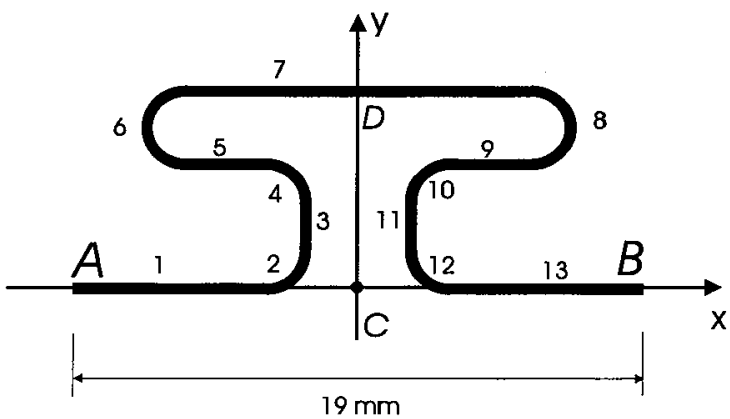

Figure 2: Orthodontic archwire: geometry data.

the maximum deformed configuration $(t=7 \mathrm{sec}$.) in combination with a contour plot for the first component of stress tensor.
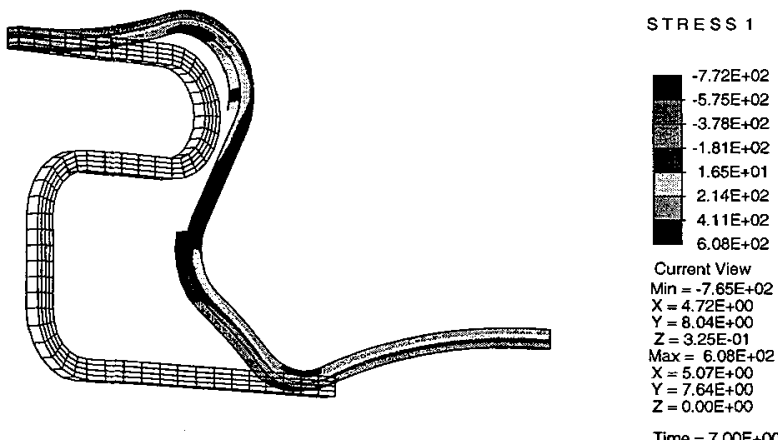

Figure 3: Orthodontic archwire: deformed mesh in the maximum deformed configuration.

\section{References}

[1] F. Auricchio and R.L. Taylor. Shape-memory alloys: modelling and numerical simulations of the finite-strain superelastic behavior. Computer Methods in Applied Mechanics and Engineering, 143:175-194, 1997.

[2] E.H. Lee. Elastic-plastic deformation at finite strains. Journal of Applied Mechanics, 36:1-6, 1969.

[3] J.C. Simo and T.J.R. Hughes. Computational inelasticity. Springer-Verlag, 1998.

[4] M.E. Gurtin. An introduction to continuum mechanics. Academic Press, 1981.

[5] F. Auricchio. A robust integration-algorithm for a finite-strain shape memory-alloy superelastic model. International Journal of Plasticity, 2000. accepted for publication. 